\title{
The contribution of hyperinsulinemia to the hyperandrogenism of polycystic ovary syndrome
}

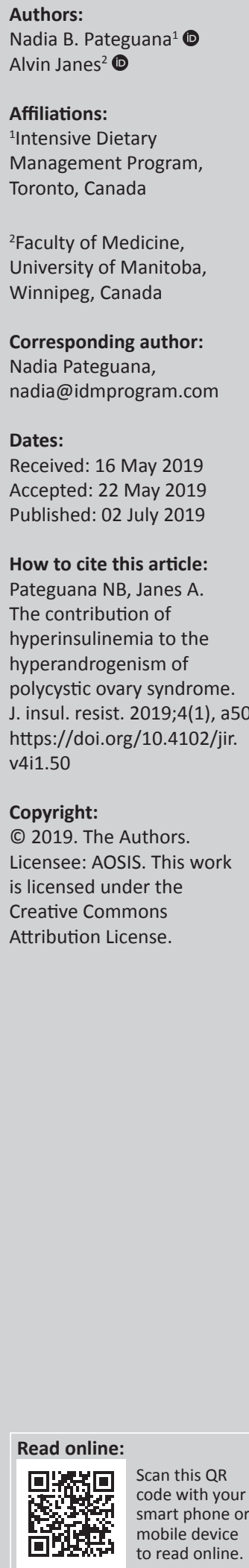

Background: Polycystic Ovary Syndrome (PCOS) is a very common endocrine disorder of young women.

Aim: The proper treatment of PCOS requires a thorough understanding of the underlying cause of disease. In this article, we review the extent to which hyperinsulinemia contributes to the development of PCOS.

Setting: The goal of this review was to assess the current literature on the contribution of hyperinsulinemia to the hyperandrogenism of polycystic ovary syndrome in hopes of promoting future research and advancements in clinical treatments for women with PCOS focusing on this major contributing factor, hyperinsulinemia.

Method: A review of published peer-reviewed literature was conducted by searching the keywords.

Results: Excessive insulin causes both the overproduction of testosterone and decreased sex hormone binding globulin (SHBG) levels seen in PCOS, both of which collaborate in creating an increased testosterone effect.

Conclusion: The majority of research and evidence shows that the hyperandrogenism of PCOS is likely caused by hyperinsulinemia. Yet the conventional treatment of hyperandrogenic symptoms in women with PCOS is not directed towards correcting this underlying hyperinsulinemia. Further research is needed to assess how the treatment of the hyperinsulinemia through lifestyle would compare to the current treatment of hyperandrogenemia through testosterone-lowering drugs.

Keywords: PCOS; hyperandrogenemia; hyperinsulinemia; fertility; insulin resistance.

\section{Introduction}

Polycystic Ovary Syndrome (PCOS) has now evolved into the most common endocrine disorder of young women, involving multiple organ systems. Newer estimates of the prevalence suggest it affects $15 \%-20 \%$ of women, making it the most common endocrine disorder of young women by far. ${ }^{1}$ The three main symptoms of PCOS are: (1) Hyperandrogenism, (2) Menstrual Irregularity and (3) Polycystic ovaries. The hormonal underpinnings of PCOS began to be appreciated in the 1950s, with the development of the radioimmunoassay that could measure various hormone levels. In the 1960s and 1970s, research focused on Luteinising Hormone (LH) and follicle stimulating hormone (FSH), key regulators of the normal menstrual cycle. For many years, an abnormal LH/FSH ratio was considered diagnostic of PCOS. ${ }^{2}$ By the 1980s, testosterone was recognised as the main androgen that was responsible for the majority of problems. ${ }^{3}$ Hyperandrogenemia is the biochemical hallmark of PCOS. ${ }^{4}$

\section{Material and methods}

The goal of this review was to assess the current literature on the contribution of hyperinsulinemia to the hyperandrogenism of polycystic ovary syndrome in hopes of promoting future research and advancements in clinical treatments for women with PCOS focusing on this major contributing factor, hyperinsulinemia.

A review of published peer-reviewed literature was conducted by searching the keywords: PCOS, polycystic ovary syndrome, polycystic ovarian syndrome, hyperandrogenemia and hyperinsulinemia. Additional articles were suggested by the authors and clinicians involved in this review. Studies were first selected based on title and abstract. A full text assessment was 
then utilised to ensure relevance. Studies were included if the title and abstract focused on the contribution of hyperinsulinemia to hyperandrogenemia.

\section{Ethical considerations}

This article followed all ethical standards for research without direct contact with human or animal subjects.

\section{Results \\ Hyperandrogenemia}

Excessive androgen production, specifically testosterone, has been extensively linked to many symptoms of PCOS. ${ }^{5}$

Testosterone is produced in both the ovaries and the adrenal glands in roughly equal proportions. ${ }^{6}$ One key question is whether the hyperandrogenism of PCOS was of ovarian or adrenal origin. For years, it was assumed that both organs were equally culpable.

By 1989, studies firmly pointed towards the ovary as the key source of excessive testosterone production in women with PCOS. Specifically, it is the theca cells within the ovary that are responsible for the overproduction of hormones. ${ }^{7}$

This suspicion is further strengthened by Drs Stein and Leventhal's research, who performed ovarian wedge resection surgery on patients with PCOS, which often restored normal ovulation and normal menstrual cycles. ${ }^{8}$ The hyperandrogenism of PCOS, secreted mostly by the ovaries, caused the symptoms of hirsutism and infertility.

Direct measurement of blood testosterone levels is not part of the diagnostic criteria for hyperandrogenemia ${ }^{9}$ and is highly problematic for three reasons. Firstly, blood testosterone levels vary widely throughout the day with age and menstrual status. ${ }^{10}$ Secondly, even in established PCOS, the ovarian portion of $T$ production only rises to about $60 \%$ of total daily production of $T .{ }^{11}$ Thirdly, a major contributing factor to the excess androgen effect seen in PCOS (hirsutism, acne etc.) is not excessive testosterone, but rather low levels of sex hormone binding globulin (SHBG). ${ }^{5}$ The SHBG is the protein designed to carry testosterone inactively; testosterone without SHBG is free to exert its effects.

Testosterone in high levels starts to exert its masculinising effects on the neighbouring organs because of the lack of $\mathrm{SHBG}^{12}$ thus the excessive development of acne, excessive facial hair, male pattern baldness and other hyperandrogenemia expressions. Women with PCOS are often found to have low levels of SHBG, ${ }^{5}$ which allows higher levels of free testosterone and greatly increases the extent of testosterone's effect on the body, even if total testosterone levels are not particularly high.

\section{Hyperinsulinemia}

Insulin and testosterone blood levels have showed a strong $85 \%$ correlation, highly suggestive that one hormone is directly stimulating the other. ${ }^{13}$ This relationship was further elucidated by in vitro studies. ${ }^{14}$ But did hyperinsulinemia stimulate testosterone production or did high testosterone stimulate hyperinsulinemia? Experimental evidence suggested that hyperinsulinemia was the primary cause. Purified ovarian cells bathed in insulin significantly increased their testosterone production. The opposite does not hold true; purified ovarian cells bathed in testosterone did not produce insulin. The pancreas is the organ responsible for insulin production and secretion, but there was little evidence that high testosterone levels, such as from a tumour, could produce hyperinsulinemia.

In vivo studies have confirmed that high insulin does indeed increase androgen levels. Direct insulin infusion measurably increases androgen production by binding to insulin or insulin-like growth factor-1 receptors in the ovary. ${ }^{15}$ The response to insulin was also graded; higher levels of insulin infusion yielded higher levels of androgen production. ${ }^{15}$ Even 24 hours after the insulin infusion was stopped, the testosterone levels continued to be elevated.

Insulin plays another role as the major regulator of SHBG production in the liver; hyperinsulinemia has been linked to hyperandrogenemia and lack of SHBG. ${ }^{16}$ Moreover, an inverse relationship between insulin levels and SHBG production has been proved for both men and women. ${ }^{17}$

Particularly with diabetics, type 1 diabetics, with very low insulin levels, have very high SHBG, and type 2 diabetics, with very high insulin levels, have very low SHBG. ${ }^{11}$

Insulin directly inhibits liver production of SHBG, and therefore increases the levels of free and bioavailable androgens. ${ }^{18}$

Thus, excessive insulin causes both the overproduction of testosterone and decreased SHBG levels, both of which collaborate in creating an increased testosterone effect.

\section{Conclusions}

The majority of research and evidence shows that the hyperandrogenism of PCOS is likely caused by hyperinsulinemia. Yet the conventional treatment of hyperandrogenic symptoms in women with PCOS is not directed towards correcting this underlying hyperinsulinemia. Rather, conventional treatments focus on lowering testosterone levels, with moderate success. This is symptomatic treatment only, and therefore would not be expected to be very successful. Instead, decreasing insulin levels through weight loss, diet and lifestyle might have greater success in overall signs and symptoms of PCOS as well as preventing associated conditions such as type 2 Diabetes, Obesity and Cardiovascular Disease.

Further research is needed to assess how the treatment of the hyperinsulinemia through lifestyle would compare to the current treatment of hyperandrogenemia through 
testosterone-lowering drugs. More research is also warranted to gain a better understanding of evidencedbased guidelines for treatment of PCOS, especially when considering specific dietary recommendations.

\section{Acknowledgements Competing interests}

The authors have declared that no competing interest exists.

\section{Authors' contributions}

N.B.P. was responsible for conceptualisation, methodology and writing. N.B.P. and A.J. were responsible for investigation. Both authors reviewed the final manuscript.

\section{Funding}

This research received no specific grant from any funding agency in the public, commercial or not-for-profit sectors.

\section{Data availability statement}

Data sharing is not applicable to this article as no new data were created or analysed in this study.

\section{Disclaimer}

The views and opinions expressed in this article are those of the authors and do not necessarily reflect the official policy or position of any affiliated agency of the authors.

\section{References}

1. Wolf WM, Wattick RA, Kinkade ON, et al. Geographical prevalence of polycystic ovary syndrome as determined by region and race/ethnicity. Int J Environ Res Public Health. 2018;15(11):2589. https://doi.org/10.3390/ijerph15112589
2. Randeva HS, Tan BK, Weickert MO, et al. Cardiometabolic aspects of the polycystic ovary syndrome. Endocr Rev. 2012;33(5):812-841. https://doi.org/10.1210/er.2012-1003

3. Cho LW, Jayagopal V, Kilpatrick ES, et al. The LH/FSH ratio has little use in diagnosing polycystic ovarian syndrome. Ann Clin Biochem. 2006;43(Pt 3): 217-219. https://doi.org/10.1258/000456306776865188

4. Rosenfield RL, Ehrmann DA. The pathogenesis of polycystic ovary syndrome (PCOS): The hypothesis of PCOS as functional ovarian hyperandrogenism revisited. Endocr Rev. 2016;37(5):467-520. https://doi.org/10.1210/er.2015-1104

5. Azziz R, Carmina E, Dewailly $D$, et al. The androgen excess and PCOS society criteria for the polycystic ovary syndrome: The complete task force report. Fertil Steril. 2009;91(2):456-488. https://doi.org/10.1016/j.fertnstert.2008.06.035

6. Pasquali R, Gambineri A. A comprehensive approach in diagnosing the polycystic ovary syndrome. Womens Health. 2015;11(4):501-512. https://doi.org/10.2217/ whe.15.13

7. Burger HG. Androgen production in women. Fertil Steril. 2002;77(4):3-5.

8. Koeppen BM, Stanton BA. Chapter 44: The male and female reproductive systems. In: Koeppen BM, Stanton BA, editors. Berne and levy physiology. 7th ed. Philadelphia, PA: Mosby/Elsevier, 2018; p. 787-829.

9. Smid I, Borsos A, Takács I. Management of female patients with the SteinLeventhal syndrome Zentralbl Gynakol.1975;97(25):1588-1593.

10. Rosenfield RL, Barnes RB, Ehrmann DA. Chapter 133: Hyperandrogenism, hirustism, and polycystic ovary syndrome. In: Larry JJ et al., editors. Endocrinology: Adult and pediatric. 7th ed. Philadelphia, PA: Saudnders/Elsevier, 2016; p. 2275-2296.

11. Cedars MI, Steingold KA, De Ziegler D, et al. Long-term administration of gonadotropin-releasing hormone agonist and dexamethasone: Assessment of the adrenal role in ovarian dysfunction. Fertil Steril. 1992;57(3):495-500.

12. Moll GW, Rosenfield RL. Testosterone binding and free plasma androgen concentrations under physiological conditions: Characterization by flow dialysis technique. J Clin Endocrinol Metab. 1979;49(5):730-736. https://doi. org/10.1210/jcem-49-5-730

13. Burghen GA, Givens JR, Kitabchi AE. Correlation of hyperandrogenism with hyperinsulinism in polycystic ovarian disease. J Clin Endocrinol Metab 1980;50(1):113-116. https://doi.org/10.1210/jcem-50-1-113

14. Willis D, Mason H, Gilling-Smith C, et al. Modulation by insulin of folliclestimulating hormone and luteinizing hormone actions in human granulosa cells of normal and polycystic ovaries. Clin Endocrinol Metab. 1996;81(1):302-309. https://doi.org/10.1210/jcem.81.1.8550768

15. Micic $D$, Popovic $V$, Nesovic $M$ et al. Androgen levels during sequential insulin euglycemic clamp studies in patients with polycystic ovary disease. J Steroid Biochem. 1988;31(6):995-999.

16. Nestler JE, Powers LP, Matt DW, et al. A direct effect of hyperinsulinemia on serum sex hormone-binding globulin levels in obese women with the polycystic ovary syndrome. J Clin Endocrinol Metab. 1991;72(1):83-89. https://doi.org/10.1210/ jcem-72-1-83

17. Strain G, Zumoff B, Rosner W, et al. The relationship between serum levels of insulin and sex hormone-binding globulin in men: The effect of weight loss. J Clin Endocrinol Metab. 1994;79(4): 1173-1176. https://doi.org/10.1210/jcem.79.4.7962291

18. Daka B, Rosen T, Jansson PA, et al. Inverse association between serum insulin and sex hormone-binding globulin in a population survey in Sweden. Endocr Connect. 2013;2(1):18-22. https://doi.org/10.1530/EC-12-0057 